\title{
Étude hydrochimique et microbiologique des eaux de source de l'ouest montagneux de la Côte d'Ivoire : Cas du village de Mangouin-Yrongouin (sous- préfecture de Biankouman).
}

\author{
${ }^{1}$ AHOUSSI Kouassi Ernest*, ${ }^{1} \mathrm{KOFFI}$ Yao Blaise, ${ }^{2} \mathrm{KOUASSI}$ Amani Michel, ${ }^{1}$ SORO Gbombélé, ${ }^{1} \mathrm{BIEMI}$ \\ Jean \\ 1. Laboratoire des Sciences et Techniques de l'Eau et de l'Environnement (LSTEE), Unité de Formation et de Recherche \\ (UFR) des Sciences de la Terre et des Ressources Minières (STRM), Université Félix Houphouët Boigny, Cocody- \\ Abidjan, 22 BP 582 Abidjan 22 ; Tel : (+225) 22483803 (Côte d'Ivoire) \\ 2. Département des Sciences de la Terre et des Ressources Minières (STeRMi), Institut National Polytechnique Félix \\ Houphouët Boigny (INP HB), BP 1093 Yamoussoukro (Côte d'lvoire), Tél. (225) 306467 15; E-mail: \\ michel.a_kouassi@yahoo.fr \\ ${ }^{*}$ Correspondance et tirés à part : ahoussi@gmx.fr \\ Original submitted in on 10 th January 2013. Published online at www.m.elewa.org on 30th March 2013.
}

\section{RÉSUMÉ}

Objectif : Évaluer la qualité physico-chimique et microbiologique des eaux de source du village de Mangouin-Yrongouin dans la localité de Biankouman (Côte d'Ivoire).

Méthodologie et résultats : Pour la réalisation de cette étude, la méthode utilisée repose sur des relevés piézométriques de la nappe phréatique, des analyses physico-chimiques et microbiologiques des eaux. Les résultats issus de ces analyses ont été traités à l'aide de techniques hydrochimiques (diagramme de Piper). L'analyse statistique multivariée dont l'Analyse en Composantes Principales (ACP) et la Classification Hiérarchique Ascendante $(\mathrm{CHA})$ a également été utilisée pour la mise en évidence des phénomènes de minéralisation des eaux de ladite localité. L'étude piézométrique montre que le niveau statique de la nappe phréatique varie de $0,48 \mathrm{~m}$ à $4,52 \mathrm{~m}$, avec une moyenne de 1,24 $\mathrm{m}$. Les eaux sont acides, avec un pH qui varie de 4,85 à 6,01. Elles sont faiblement minéralisées, avec une conductivité électrique moyenne de 152,90 $\mu \mathrm{S} / \mathrm{cm}$. Dans la zone d'étude, les eaux sont chlorurées calciques. Les analyses chimiques révèlent que les eaux de Mangouin-Yrongouin sont dépourvues d'iode. L'analyse des métaux lourds montre qu'en dehors du fer et du Ni qui présentent des teneurs supérieures à la valeur guide OMS qui est respectivement de $0,3 \mathrm{mg} / \mathrm{L}$ et $0,07 \mathrm{mg} / \mathrm{L}$, les autres métaux étudiés sont pratiquement inexistants dans les eaux. Sur le plan microbiologique, les eaux de source contiennent de forts taux d'Escherichia coli (400 à 500 ufc/100 ml) et de Clostridium perfringens ( $100 \mathrm{ufc} / 20 \mathrm{ml}$ à $1500 \mathrm{ufc} / 20 \mathrm{ml}$ ). La minéralisation des eaux est contrôlée par les phénomènes tels que le pluviolessivage des sols, l'hydrolyse acide des roches et l'apport lié aux activités anthropiques.

Conclusion et applications : Cette étude montre que les ressources en eau de Mangouin-Yrongouin sont soumises à une pression anthropique importante. La consommation de ces eaux en dehors de tout traitement présente des risques sanitaires pour les populations.

Mot clés : Assainissement, eaux de source, pollution, Hydrochimie, Environnement, Éléments Traces Métalliques. 


\section{Ahoussi et al... J. Appl. Biosci. 2013. Étude hydrochimique et microbiologique des eaux de source de l'ouest montagneux, Cote d'Ivoire}

\section{INTRODUCTION}

La croissance démographique que connaît la Côte d'Ivoire après sont indépendance a eu pour conséquence l'augmentation des besoins primaires des populations dont l'accès à l'eau potable des populations urbaines et rurales. Cette grande pression démographique sur les ressources naturelles se traduit par une production de plus en plus importante de déchets de toute nature. Ainsi, depuis ces dernières décennies, les études réalisées sur les ressources en eau de la Côte d'Ivoire par Ahoussi et al. (2008, 2009, 2010 et 2012a), Coulibaly et al. (2008) et Soro et al. (2010) ont signalé de nombreux foyers de pollution aussi bien au niveau des eaux de surface que des eaux souterraines, dans les localités d'Abidjan, de Kossihouen et de N'zianouan. Le village de Mangouin-Yrongouin situé dans le département de Biankouman n'est pas en marge de ces problèmes environnementaux. Ladite localité objet de cette étude est défavorisée par l'inexistence d'un système d'assainissement adéquat. On y observe par endroits la présence de tas d'ordures à plusieurs endroits. Les effluents de cette localité sont généralement déversés dans la nature. Les

\section{PRÉSENTATION DE LA ZONE D'ÉTUDE}

Le village Mangouin-Yrongouin est situé dans le département de Biankouma à l'Ouest de la Côte d'Ivoire ; précisément à $32 \mathrm{~km}$ de la ville de Man et $13 \mathrm{~km}$ de Biankouma, sur l'axe routier ManBiankouma à la longitude $07^{\circ} 38^{\prime} 23,8^{\prime \prime}$ Ouest et la latitude $07^{\circ} 38^{\prime} 38,4^{\prime \prime}$ Nord. Cette localité abrite une population estimée à 5000 habitants. Cette population est constituée en majorité de Yacouba, Mahouka; Toura, et les Senoufo en ce qui concerne les autochtones et de Guinéens, Burkinabés et Maliens pour les allogènes. Le village de MangouinYrongouin qui appartient à la région de Man tire son originalité par un paysage de montagne à altitude élevée, dépassant 1000 mètres. Le massif de Man est plus étalé et le moins élevé. II est constitué d'une succession d'inselbergs séparés par des vallées populations suite aux manquantes de toilettes font leur besoins dans la nature. En saison de pluies, les eaux de ruissellement, érodent les sols et transportent tous les déchets du sol ainsi les selles jusqu'aux eaux de sources. Dans ladite l'eau potable est une denrée rare. En effet, le forage d'eau d'alimentation en eau du village est en panne depuis plusieurs années. Cette situation a conduit les populations à utiliser l'eau de source comme principal point d'alimentation. La consommation de ces eaux sans traitement expose les populations à des risques sanitaires. Ainsi dans cette localité, une enquête réalisée sur le terrain lors de la réalisation de ces travaux a montré que les maladies diarrhéiques liées à l'absence d'eau potable sont récurrentes. Cette étude a été initiée pour évaluer l'influence des activités anthropiques sur la qualité des ressources en eau du village de MangouinYrongouin. Elle s'appuie sur l'utilisation des données piézométriques, hydrochimiques et microbiologiques pour la caractérisation de la pollution des eaux et des phénomènes à l'origine de la dégradation de la qualité des eaux de cette localité.

profondes et s'étend sur plus d'une centaine de kilomètres d'Est en Ouest. Le massif de Man est formé de deux grands blocs de parts et d'autres de l'incision de la vallée du N'zo: (i) les monts Toura à l'Est qui sont nus; et (ii) les monts Dan à l'Ouest couverts par la forêt. L'ensemble du massif de Man présente des altitudes variant de 500 à plus de 1000 mètres et des pentes fortes. Ailleurs, des pointements moins hauts sont remarquables par leurs formes beaucoup plus agressives, où l'on reconnaît de véritables pics plus au moins déchiquetés telle la dent de Man (881 mètres). L'aspect de ce relief fait de Man la région la plus « accidentée » du pays. 


\section{Ahoussi et al... J. Appl. Biosci. 2013. Étude hydrochimique et microbiologique des eaux de source de l'ouest montagneux, Cote d'Ivoire}

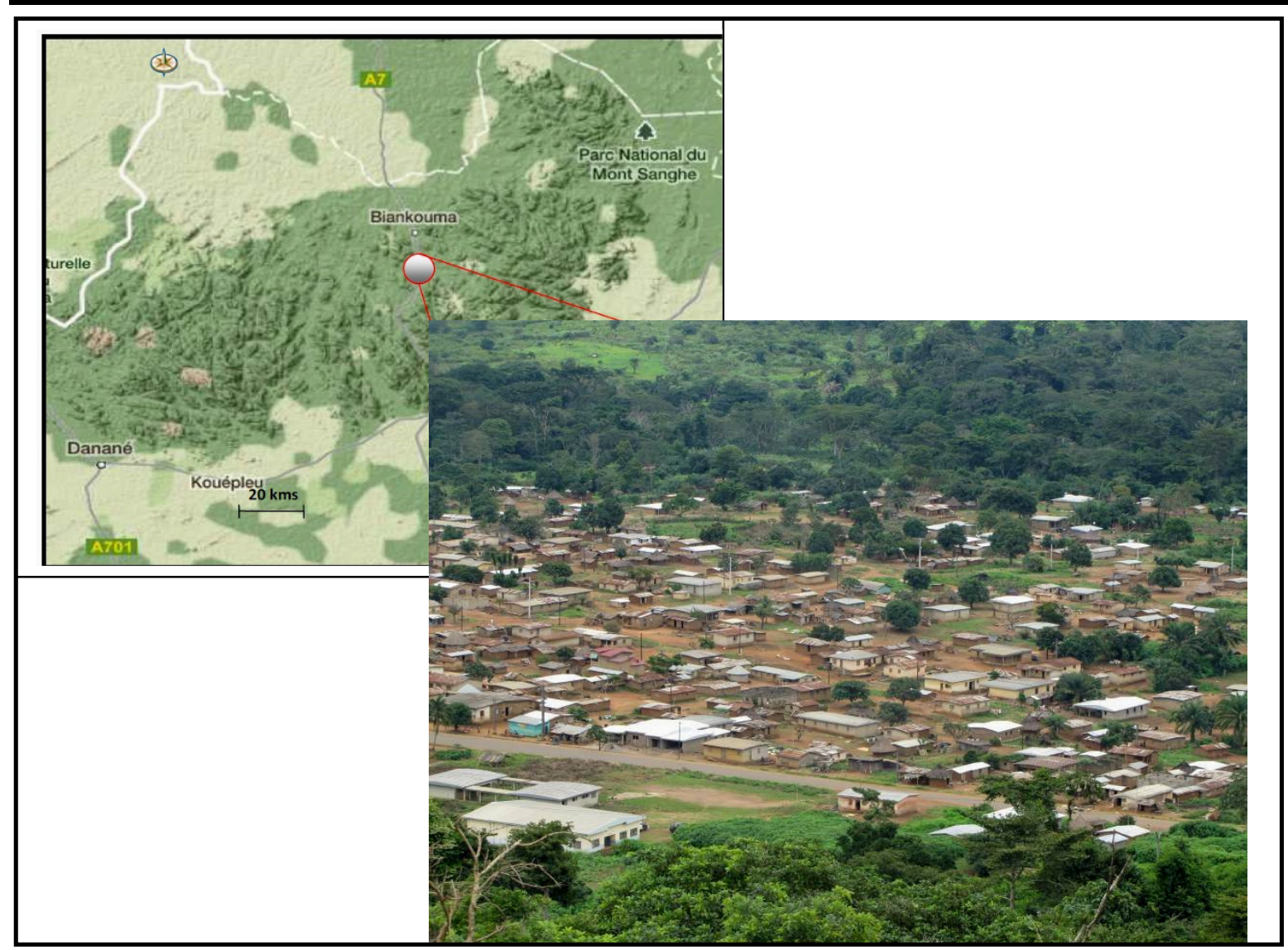

Figure 1. Localisation géographique du village de Mangouin-Yrongouin

Cadre géologique de la localité de la zone d'étude: La localité de Mangouin-Yrongouin appartient à la région de Man-Biankouma qui est située dans le domaine libérien de la dorsale de Man (Figure 2). Cette dorsale se poursuit vers l'Est par des formations archéennes du Libéria et de la Sierra Léone. Elle est limitée dans sa partie orientale par la faille du Sassandra, frontière entre les domaines libériens et éburnéens. Les études minéralogiques, métamorphiques et géochronologiques entreprises par Kouamélan (1996) ont permis d'établir l'histoire évolutive de la région. Sur le plan lithologique, trois domaines ont été identifiés. II s'agit du domaine granulite Nord; du domaine granulite et magmatique intermédiaire; du domaine sud des gneiss migmatitiques à biotite. La figure 2 montre les principales unités géologiques de la région de Biankouman et de ses environs. Les travaux de Camil et al. (1984) ont permis de recenser dans l'ordre chronologique les différentes unités lithologiques constituées d'un ensemble de gneiss gris. II est d'un aspect rubanné et d'origine magmatique; un ensemble de quarzites à magnétites: il est essentiellement d'origine sédimentaire et comportant diverses formations associées (diopsidites et hypersthinites); les charnockites intrusives : très développées dans le compartiment Nord. Du point de vue structural, la faille du Sassandra, limite entre le domaine libérien et éburnéen est identifiée selon des études de Djro (1998), comme étant une succession des phases tectoniques, séparées par des périodes de "calme" relatif. II identifie quatre phases de déformation accompagnées chacune d'un degré de métamorphisme différent: une première phase qui est un aplatissement ; un cisaillement ductile, dextre de direction $\mathrm{N} 160$; une transgression et un cisaillement fragile. 


\section{Ahoussi et al... J. Appl. Biosci. 2013. Étude hydrochimique et microbiologique des eaux de source de l'ouest montagneux, Cote d'Ivoire}

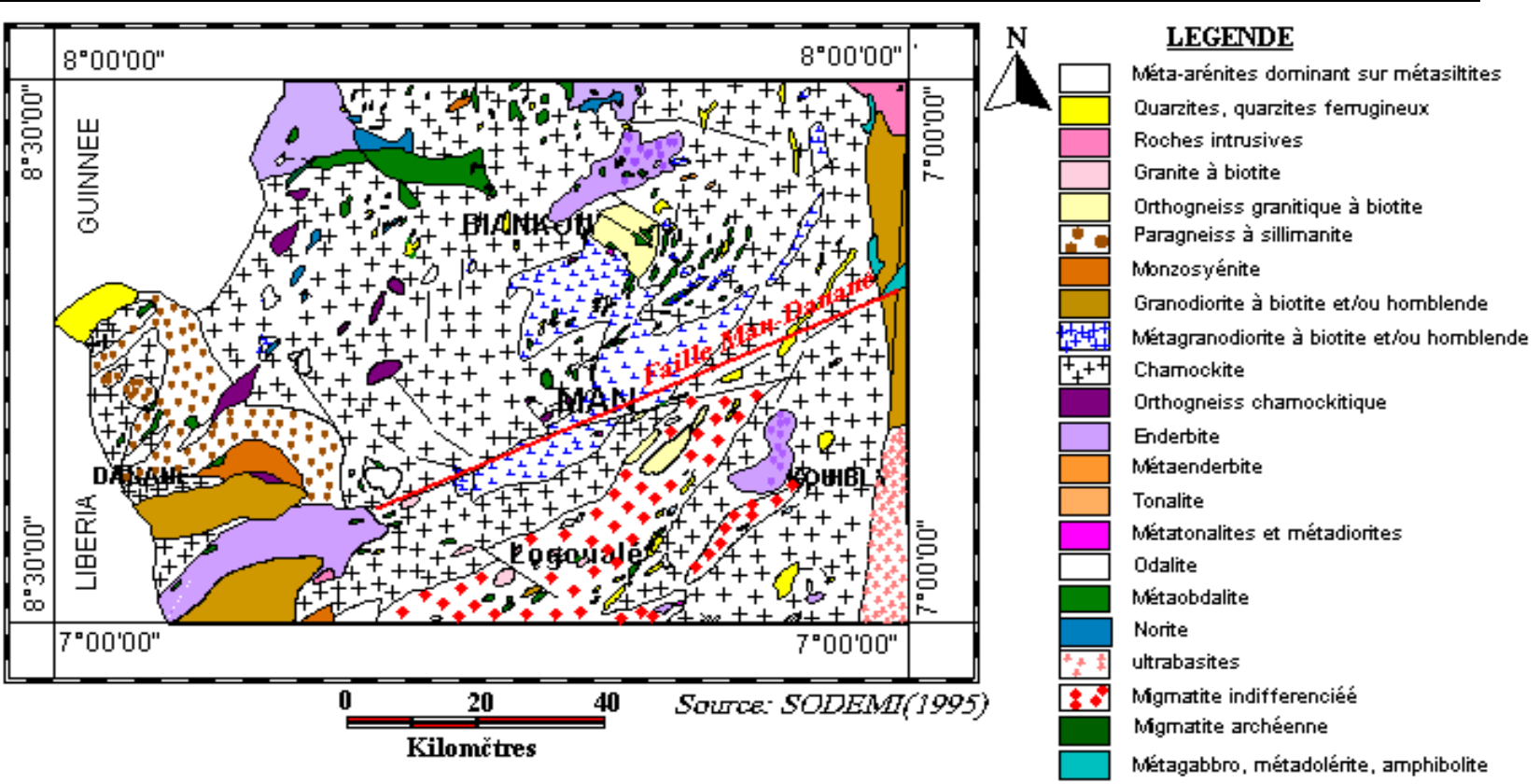

Figure 2 : Carte géologique de la région de Man-Biankouman (Saley, 2003)

Cadre hydrogéologique et pédologique de Mangouin-Yrongouin : Dans la zone d'étude, les aquifères qui s'y trouvent se composent de trois réservoirs superposés. En haut, se trouve les altérites qui sont composées de cuirasse latéritique, d'argile, d'arène lorsque les formations géologiques sont de type granitoïde. La zone du milieu est formée d'éléments rocheux et de produits d'altération, avec la présence ou non de nombreuses fissures. Cette zone, lorsqu'elle contient de l'eau, constitue le principal réservoir de l'aquifère de socle. Le troisième réservoir se situe dans le socle fracturé et est parcouru par de simples fractures ou dans certains cas par des zones broyées dues à des contraintes locales assez fortes. Ce réservoir de socle est souvent le siège de circulation d'eau souterraine. Les trois réservoirs sont en fait considérés comme un système aquifère bicouche formé d'un réservoir semi-perméable capacitif (les altérites) surmontant un réservoir, formé de roche fissurée, captif à fonction essentiellement conductrice. L'aquifère des altérites possède une porosité totale très élevée mais une porosité efficace très faible engendrant des perméabilités faibles sauf pour les parties superficielles fortement lessivées. Ces aquifères sont captés dans le village par des puits fournissant des débits d'exploitation et des débits spécifiques faibles. La qualité de l'eau est médiocre à cause des nombreux minéraux argileux et du risque de pollution des eaux depuis la surface. Cependant, les ressources en eau de la nappe phréatique sont très variables à cause de la variabilité verticale et latérale des caractéristiques hydrogéologiques essentielles. Ces aquifères reçoivent directement les eaux des précipitations et leurs niveaux piézométriques baissent considérablement en saison sèche et remontent en saison de pluie (Biémi, 1992). La nappe phréatique est plus facilement accessible à travers les puits modernes, les puits traditionnels ou les puisards temporaires creusés dans les bas-fonds pendant la saison sèche. Malgré une pluviométrie importante, l'environnement fait que le coefficient de ruissellement est très élevé. Les sols rencontrés dans cette région sont de types ferralitiques. Compte tenu du caractère très accidenté de la zone, ces sols ont subi un lessivage intense, suite à l'importante pluviométrie de la région (3000 mm à Danané, 2000 $\mathrm{mm}$ à Man et environs). Les sols à valeur agronomique moyenne constituent $12 \%$ des sols de la zone d'étude. Les sols riches et profonds, de texture argileuse qui convient à la culture du cacao, ne représentent que $5 \%$ des sols. 


\section{Ahoussi et al... J. Appl. Biosci. 2013. Étude hydrochimique et microbiologique des eaux de source de l'ouest montagneux, Cote d'Ivoire}

\section{MATÉRIEL ET MÉTHODES}

Méthode d'étude piézométrique : Une campagne de relevés piézométriques a été organisée en août 2012 dans le village de Mangouin-Yrongouin pour la mesure du niveau statique de la nappe phréatique dans les différents puits villageois. Cette étude a démarré par le repérage de tous les puits du village à partir d'un appareil GPS (Global Positioning System). Les mesures piézométriques ont été réalisées à l'aide d'une sonde piézométrique OTT (100 $\mathrm{m})$ sonore et lumineuse. Les données collectées sur le terrain ont permis de déterminer la côte des eaux souterraines selon la formule suivante :

$$
\mathrm{C}_{\text {eau }}=\mathbf{Z}-\mathrm{H}
$$

avec $\quad Z$ : altitude du piézomètre $(m)$;

$H=(N P+M)$ : profondeur de l'eau dans le piézomètre $(\mathrm{m})$;

$N P$ : niveau piézométrique $(\mathrm{m})$;

$M$ : margelle des puits ;

$\mathrm{C}_{\text {eau }}$ : côte de l'eau $(\mathrm{m})$.

Pendant la campagne de relevés piézométriques, la profondeur des puits a également été mesurée. La lame d'eau dans le puits a été déterminée par la formule suivante :

$$
L_{\text {eau }}=\text { Prof (puits) }- \text { NP }
$$

avec Leau: lame d'eau en $(m)$;

$$
\text { Prof (puits) : profondeur du puits en }(\mathrm{m}) \text {; }
$$

Méthode d'étude hydrochimique et microbiologique Méthode d'échantillonnage: L'échantillonnage des eaux du village de Mangouin-Yrongouin a été effectué en août 2012 pendant la petite saison sèche. II a porté sur les eaux de sources, les eaux de puits et les eaux de surface. Dans la zone d'étude, nous avons identifié six (6) sources d'eau dont trois (3) sources d'eau pérennes qui se situent dans les environs immédiats du village. Parmi celles-ci, trois sont utilisées par la population du village pour les besoins en eau potable (Figure 3 et 4 ) en remplacement de l'eau courante. En plus des sources d'eau utilisées pour l'alimentation en eau, les populations s'alimentent également à partir des puits. Les eaux de puits sont généralement utilisées pour la lessive, la vaisselle et pour la boisson

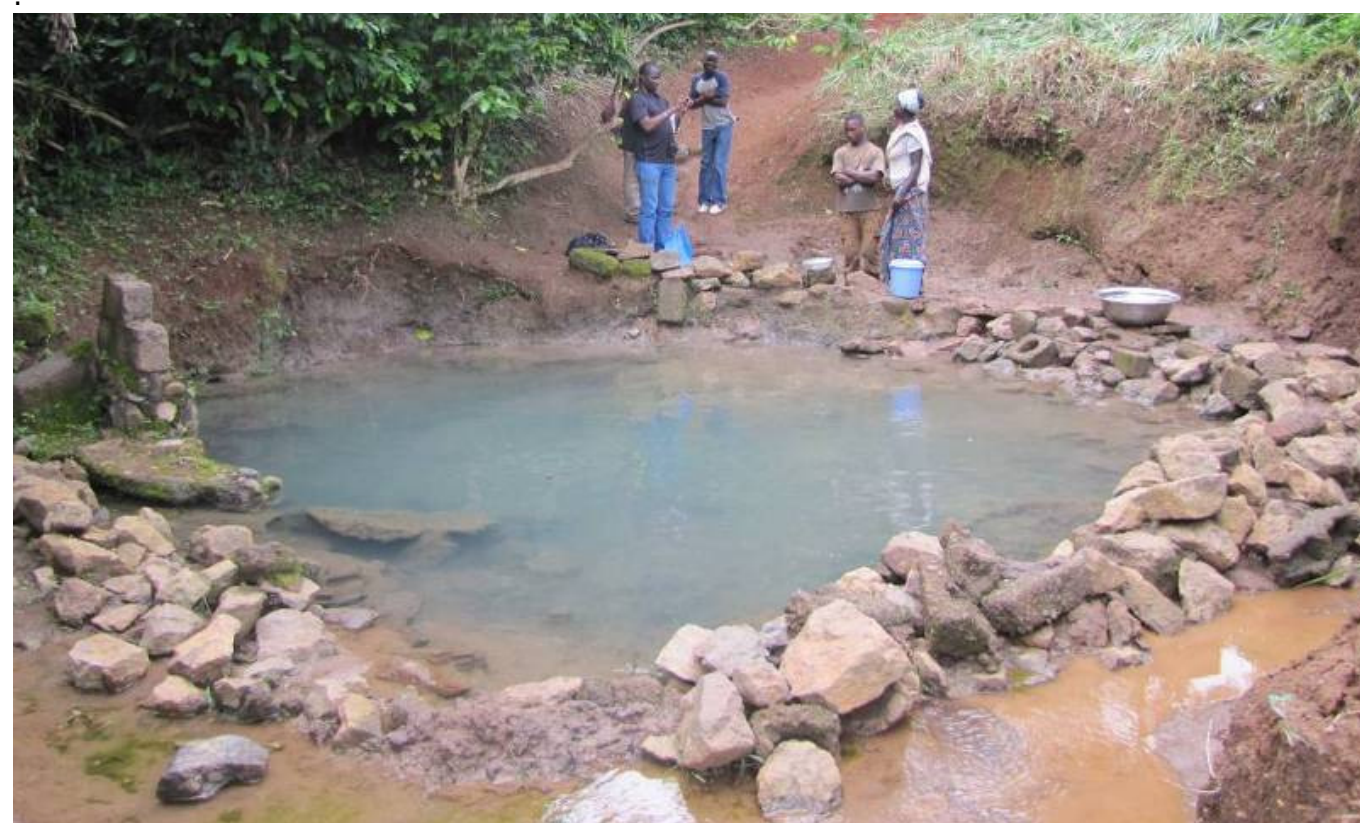

Figure 3 : Site d'alimentation en eau principale de la population de Mangouin pour ses besoins domestiques 


\section{Ahoussi et al... J. Appl. Biosci. 2013. Étude hydrochimique et microbiologique des eaux de source de l'ouest montagneux, Cote d'Ivoire}

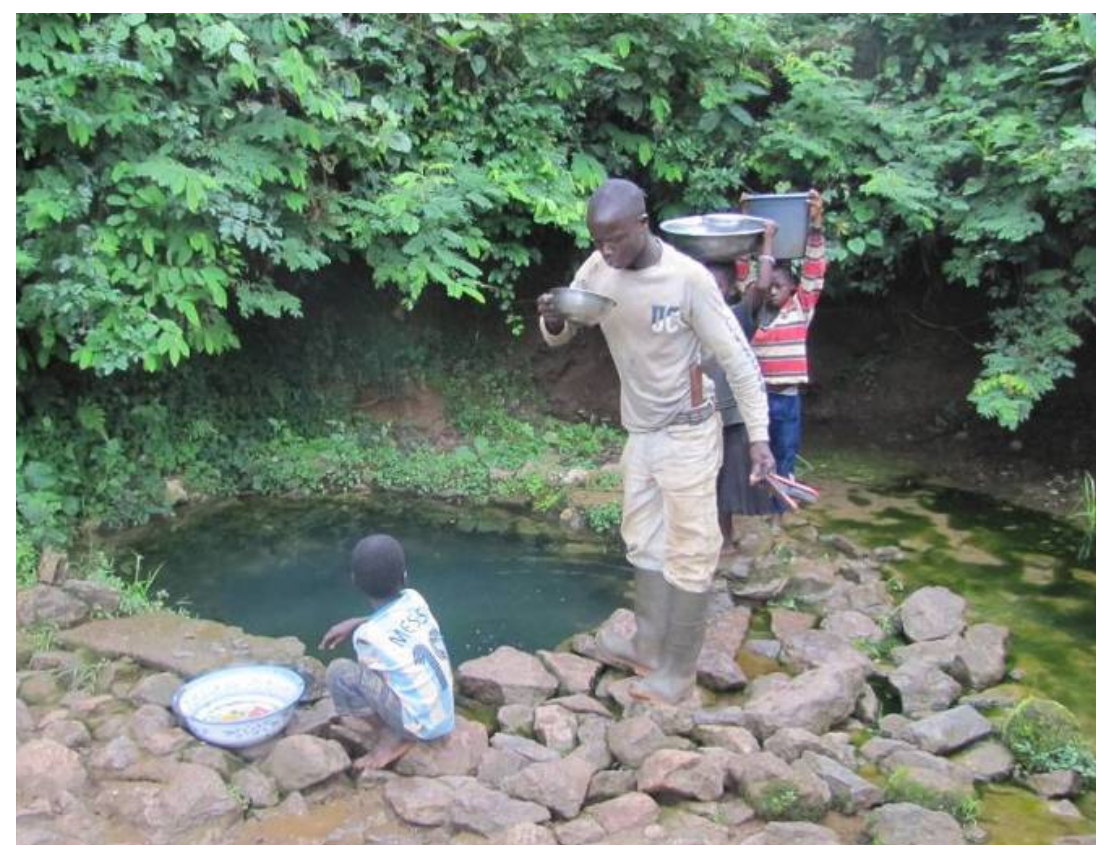

Figure 4 : Site d'approvisionnement en eau potable de la population d'Yrongouin

Lors de cette campagne, les échantillons d'eau prélevés ont été mis dans des bouteilles en polyéthylène de capacité $500 \mathrm{ml}$ et 1 litre, préalablement lavées à l'acide nitrique puis à l'eau distillée. Sur le terrain, avant le remplissage des bouteilles, celles-ci ont été lavées trois fois avec l'eau à prélever. Le remplissage des bouteilles a été fait à ras bord puis le bouchon vissé afin d'éviter tout échange gazeux avec l'atmosphère. Les échantillons d'eau ont été ensuite transportés dans une glacière à 4 ${ }^{\circ} \mathrm{C}$ au laboratoire pour analyse dans l'heure qui suit le prélèvement. Lors des prélèvements, les paramètres physiques des eaux tels que le $\mathrm{pH}$, la conductivité électrique (CE) et le potentiel d'oxydoréduction (Eh) ont été mesurés in situ. L'appareillage utilisé sur le terrain est constitué d'un pH-mètre Star 4 pour la mesure du pH et Eh, d'un conductimètre Hach Sension 5 pour la mesure de la conductivité électrique (CE) et de la salinité (Sal.) Les eaux prélevées ont fait l'objet de plusieurs analyses dont les analyses physico-chimiques et microbiologiques. Les éléments chimiques dosés sont: calcium $\left(\mathrm{Ca}^{2+}\right)$, magnésium $\left(\mathrm{Mg}^{2+}\right)$, sodium $\left(\mathrm{Na}^{+}\right)$, potassium $\left(\mathrm{K}^{+}\right)$, chlorure $(\mathrm{Cl})$, sulfate $\left(\mathrm{SO}_{4}{ }^{2}\right)$ et bicarbonate $\left(\mathrm{HCO}_{3}{ }^{-}\right)$, pour ions majeurs et nitrate $\left(\mathrm{NO}_{3}{ }^{-}\right)$, nitrite $\left(\mathrm{NO}_{2}{ }^{-}\right)$, ammonium $\left(\mathrm{NH}_{4}{ }^{+}\right)$, phosphate $\left(\mathrm{PO}_{4}^{3-}\right)$ pour les sels nutritifs. Les métaux tels que cuivre $(\mathrm{Cu})$, plomb $(\mathrm{Pb})$, chrome $(\mathrm{Cr})$ et arsenic (As) ont également fait l'objet d'analyse. Aussi, la Demande Biologique en Oxygène sur 5 jours ( $\mathrm{DBO}_{5}$ ), la Demande Chimique en Oxygène (DCO) et l'iode des eaux ont-elles été également mesurées. Les échantillons d'eau pour analyse microbiologique ont été prélevés dans des bouteilles de $500 \mathrm{ml}$, préalablement stérilisées et conservés dans une glacière à $4^{\circ} \mathrm{C}$. Les analyses microbiologiques ont concerné les bactéries telles qu'Escherichia coli, Clostridiums Perfringens et les coliformes totaux. Les échantillons d'eau ont été prélevés sur les principaux points d'eau du village. II s'agit des eaux de sources, du lac de la société GRANOCl et des puits du village.

Méthode d'analyse et de traitement des données : Au laboratoire, les cations majeurs, ont été dosés par la méthode de spectrophotométrie à l'aide d'un spectrophotomètre de type AAS VARIAN. Les sels nutritifs ont été analysés à partir de la méthode de spectrophotométrie par un spectrophotomètre de type UV SHIMADZU. Les bicarbonates ont été dosés à partir de la méthode de dosage à l'aide d'un titrateur digital. Les chlorures ont été mesurés par la méthode de Mohr par titrage. Les sulfates, la DCO ont été dosés par spectrophotométrie à l'aide d'un spectrophotomètre HACH DR 2010. La DBO 5 a été dosée par incubation à partir d'un incubateur de type $\mathrm{HACH}$. La turbidité des eaux a été mesurée à partir d'un turbidimètre 2020. Les métaux ont été dosés par spectrométrie à l'aide d'un spectrophotomètre à absorption atomique VARIAN. Les analyses microbiologiques ont nécessité l'utilisation de méthodes selon les microorganismes recherchés. Ainsi, pour le dénombrement des coliformes totaux, nous avons fait appel à la méthode NF V08-50 et pour le 


\section{Ahoussi et al... J. Appl. Biosci. 2013. Étude hydrochimique et microbiologique des eaux de source de l'ouest montagneux, Cote d'Ivoire}

dénombrement des coliformes fécaux de type Eschérichia, la méthode AFNOR BRD 07/1-07/93 a été utilisée. Les données collectées sur le terrain et au laboratoire ont été traitées à partir des méthodes statistiques mutlivariées couplées aux méthodes hydrochimiques. La méthode hydrochimique a nécessité l'utilisation du diagramme de Piper pour la classification hydrochimique des eaux. Ce diagramme est très fréquemment utilisé dans le domaine de hydrochimie, avec de très bons résultats, par Kouzana et al. (2007), Oga et al. (2009), Ahoussi et al. (2010, 2012b). L'approche statistique est basée sur l'utilisation de l'Analyse en Composantes Principale (ACP) et la Classification Hiérarchique Ascendante ( $\mathrm{CHA}$ ) pour l'étude des phénomènes à l'origine de la minéralisation

\section{RÉSULTATS ET DISCUSSION}

Résultats Hydrogéologiques: Les résultats de la campagne de relevés piézométrique sont consignés dans le tableau 1. La profondeur de la nappe phréatique varie de $0,48 \mathrm{~m}$ à 4,52 $\mathrm{m}$, avec une moyenne de $1,24 \mathrm{~m}$, ce qui montre que la nappe phréatique est peu profonde. La lame d'eau dans les puits varie de $0,29 \mathrm{~m}$ à $4,52 \mathrm{~m}$, avec une moyenne de 1,90 m.

Résultats de l'étude physico-chimique: Les sites d'échantillonnage des eaux souterraines, des eaux de source et des eaux de surface sont présentés dans le tableau 2. Les principaux points d'eau échantillonnés font l'objet de site d'alimentation en eau pour les populations villageoises.

Résultats des mesures in situ: Les paramètres physiques des eaux sont consignés dans le tableau 3. A l'analyse de celui-ci, il ressort que les eaux sont acides, des eaux. Ces deux méthodes statistiques sont couramment utilisées dans le domaine des sciences de l'eau, avec d'excellents résultats dans l'étude de l'origine des minéralisations des eaux, par les auteurs tels Güler et al. (2002) ; Yidana (2008) ; Ahoussi et al. (2012a) ; Thilagavathi et al. (2012). Les analyses ont été effectuées à partir de 21 variables qui sont : $\mathrm{pH}$, Turbidité (Turb), Eh, Sal., $\mathrm{CE}, \mathrm{HCO}_{3}^{-}, \mathrm{SO}_{4}{ }^{2-}, \mathrm{Cl}-\mathrm{Na}^{+}, \mathrm{K}^{+}, \mathrm{Ca}^{2+}, \mathrm{Mg}^{2+}, \mathrm{NO}_{3}{ }^{-}$, $\mathrm{NO}_{2}-\mathrm{NH}_{4}{ }^{+}, \mathrm{Fe}, \mathrm{Mn}, \mathrm{Pb}, \mathrm{Cu}, \mathrm{Cr}$ et $\mathrm{Pb}$. Les analyses statistiques ont été réalisées sous le logiciel STATISTICA 6.0 . Les résultats des analyses microbiologiques ont été également comparés aux valeurs guides de l'Organisation Mondiale de Santé (OMS, 2008) dans le cadre d'une eau de boisson, non influencée par les activités anthropiques.

avec un pH qui varie de 4,85 à 6,01 , pour une moyenne de 5,34. La conductivité électrique des eaux source du village varie de 112,30 à $178,20 \mu \mathrm{S} / \mathrm{cm}$, avec une moyenne de 152,90 $\mu \mathrm{S} / \mathrm{cm}$. Ces eaux sont faiblement minéralisées. Dans la zone, les conductivités sont graduelles, elles évoluent respectivement de $62,9 \mu \mathrm{S} / \mathrm{cm}$ pour l'eau du lac (eau de surface) à $304 \mu \mathrm{S} / \mathrm{cm}$ pour l'eau de puits. Les puits présentent les conductivités les plus élevées. Les eaux de source ont une turbidité qui varie de 0,98 à 10,01 NTU, avec une moyenne de 4,08 NTU. Les eaux ont un potentiel d'oxydoréduction positif qui varie de $35 \mathrm{mv}$ à $100 \mathrm{mv}$, avec une moyenne de $73 \mathrm{mv}$. La DCO varie de 58 à $80 \mathrm{mg} / \mathrm{L}$ (moyenne $72 \mathrm{mg} / \mathrm{L}$ ). Quant au $\mathrm{DBO}_{5}$, elle varie de $23 \mathrm{mg} / \mathrm{L}$ à $38 \mathrm{mg} / \mathrm{L}$, avec une moyenne de $31 \mathrm{mg} / \mathrm{L}$. 


\section{Ahoussi et al... J. Appl. Biosci. 2013. Étude hydrochimique et microbiologique des eaux de source de l'ouest montagneux, Cote}

d'Ivoire

Tableau 1 : Récapitulatif des données piézométriques et des caractéristiques des ouvrages hydrauliques

\begin{tabular}{|c|c|c|c|c|c|c|c|c|c|}
\hline $\mathbf{N}^{\circ}$ & Site & Ouvrage & Latitude & Longitude & Alt. (m) & Niv. Eau & Prof (m) & Côte Eau (m) & Lame d'eau (m) \\
\hline 1 & Mangouin & Puits & $07^{\circ} 39^{\prime} 13,9^{\prime \prime} \mathrm{N}$ & $07^{\circ} 49^{\prime} 17,5^{\prime \prime} \mathrm{W}$ & 581 & 3,87 & 6,05 & 577,13 & 2,18 \\
\hline 2 & Mangouin & Puits & $07^{\circ} 38^{\prime} 38,4^{\prime \prime} \mathrm{N}$ & $07^{\circ} 38^{\prime} 23,8^{\prime \prime} \mathrm{W}$ & 587 & 12,1 & 13,98 & 574,9 & 1,88 \\
\hline 3 & Mangouin & Puits & $07^{\circ} 38^{\prime} 35,5^{\prime \prime} \mathrm{N}$ & $07^{\circ} 36^{\prime} 23,1^{\prime \prime} \mathrm{W}$ & 581 & 5,58 & 10,1 & 575,42 & 4,52 \\
\hline 4 & Mangouin & puits & $07^{\circ} 38^{\prime} 34,5 " \mathrm{~N}$ & $07^{\circ} 36^{\prime} 21,8^{\prime \prime W}$ & 580 & 7,01 & 7,51 & 572,99 & 0,5 \\
\hline 5 & Mangouin & Puits & $07^{\circ} 38^{\prime} 33,2 " \mathrm{~N}$ & $07^{\circ} 36^{\prime} 20,7^{\prime \prime} \mathrm{W}$ & 578 & 6,25 & 8,97 & 571,75 & 2,72 \\
\hline 6 & Mangouin & Source de Gahiba $N^{\circ} 5$ & $07^{\circ} 38^{\prime} 29,2 " \mathrm{~N}$ & $07^{\circ} 36^{\prime} 20,2^{\prime \prime W}$ & 567 & - & - & - & - \\
\hline 7 & Mangouin & Source $N^{\circ} 6$ & $07^{\circ} 38^{\prime} 26,30^{\prime \prime} \mathrm{N}$ & $07^{\circ} 36^{\prime} 22,5^{\prime \prime} \mathrm{W}$ & 572 & - & - & - & - \\
\hline 8 & Mangouin & Puits & $07^{\circ} 38^{\prime} 26,30^{\prime \prime} \mathrm{N}$ & $07^{\circ} 36^{\prime} 24,8^{\prime \prime} \mathrm{W}$ & 582 & 8,19 & 10,37 & 573,81 & 2,18 \\
\hline 9 & Mangouin & Puits & $07^{\circ} 38^{\prime} 27,8 " \mathrm{~N}$ & $07^{\circ} 36 ' 26,7^{\prime \prime W}$ & 586 & 7,51 & 9,72 & 578,49 & 2,21 \\
\hline 10 & Mangouin & Puits & $07^{\circ} 38^{\prime} 26,6^{\prime \prime} \mathrm{N}$ & $07^{\circ} 36 ' 27,8^{\prime \prime} \mathrm{W}$ & 586 & 7,88 & 8,17 & 578,12 & 0,29 \\
\hline 11 & Mangouin & Puits & $07^{\circ} 38^{\prime} 30,1 " \mathrm{~N}$ & $07^{\circ} 36 ' 28,8^{\prime \prime W}$ & 590 & 8,48 & 8,85 & 581,52 & 0,37 \\
\hline 12 & Mangouin & Puits & $07^{\circ} 38^{\prime} 32,5^{\prime \prime N}$ & $07^{\circ} 36^{\prime} 29^{\prime \prime W}$ & 591 & 6,33 & 8,43 & 584,67 & 2,1 \\
\hline
\end{tabular}

Tableau 2. Sites de prélèvement des eaux de Mangouin-Yrongouin

\begin{tabular}{c|c|l|l|l|l|l}
\hline $\mathbf{N}^{\circ}$ & $\begin{array}{c}\text { Sites de } \\
\text { prélèvement }\end{array}$ & Ouvrages & Latitude & Longitude & Alt. $(\mathrm{m})$ & Lame d'eau $(\mathrm{m})$ \\
\hline E1 & Mangouin & Source 1 & $07^{\circ} 38^{\prime} 43,3^{\prime \prime} \mathrm{N}$ & $07^{\circ} 36^{\prime} 33,35^{\prime \prime} \mathrm{W}$ & 579 & Prise d'eau principale du village : eau utilisée pour la boisson \\
\hline E2 & Mangouin & Lac : eau de surface & $07^{\circ} 38^{\prime} 38,9^{\prime \prime} \mathrm{N}$ & $07^{\circ} 36^{\prime} 34,3^{\prime \prime} \mathrm{W}$ & 571 & $\begin{array}{l}\text { Lac de Granoci : eau de surface. Non utilisée pour les besoins } \\
\text { des populations. Ecoulement N-S }\end{array}$ \\
\hline E3 & Mangouin & Source 2 & $07^{\circ} 38^{\prime} 50,8^{\prime \prime} \mathrm{N}$ & $07^{\circ} 36^{\prime} 22^{\prime \prime} \mathrm{W}$ & 569 & Eau de source clôturée et utilisée pour l'AEP \\
\hline E4 & Mangouin & Puits & $07^{\circ} 38^{\prime} 26,30^{\prime \prime} \mathrm{N}$ & $07^{\circ} 36^{\prime} 24,8^{\prime \prime} \mathrm{W}$ & 582 & Près de la cours du chef de village \\
\hline E5 & Yrongouin & Source 4 & $07^{\circ} 38^{\prime} 26,3^{\prime \prime} \mathrm{N}$ & $07^{\circ} 36^{\prime} 22,5^{\prime \prime} \mathrm{W}$ & 572 & $\begin{array}{l}\text { Source à usage domestique, mal entretenue avec présence } \\
\text { d'algue, eau stagnante }\end{array}$ \\
\hline
\end{tabular}




\section{Ahoussi et al... J. Appl. Biosci. 2013. Étude hydrochimique et microbiologique des eaux de source de l'ouest montagneux, Cote d'Ivoire}

Tableau 3. Récapitulatif des mesures des paramètres physiques et chimiques des eaux de source de MangouinYrongouin

\begin{tabular}{|c|c|c|c|c|}
\hline Paramètres physico-chimiques & $\begin{array}{l}\text { Valeur Guide } \\
\text { OMS (2008) }\end{array}$ & Moyenne & Min & Max \\
\hline \multicolumn{5}{|l|}{ Paramètres physiques } \\
\hline $\mathrm{pH}$ & $6-8,5$ & 5,34 & 4,85 & 6,01 \\
\hline $\mathrm{Eh}(\mathrm{mv})$ & - & 73,20 & 35,40 & 100,00 \\
\hline Cond $(\mu S)$ & - & 152,90 & 112,30 & 178,20 \\
\hline Sal \% & - & 0,00 & 0,00 & 0,00 \\
\hline Turb (NTU) & - & 4,08 & 0,98 & 10,01 \\
\hline $\mathrm{DCO}(\mathrm{mg} / \mathrm{L})$ & - & 72,00 & 58,00 & 80,00 \\
\hline $\mathrm{DBO}_{5}(\mathrm{mg} / \mathrm{L})$ & - & 31,00 & 23,00 & 38,00 \\
\hline \multicolumn{5}{|l|}{ Paramètres chimiques en $\mathrm{mg} / \mathrm{L}$} \\
\hline $\mathrm{HCO}_{3}^{-}$ & - & 68,77 & 53,70 & 80,00 \\
\hline $\mathrm{Cl}^{-}$ & 250 & 234,00 & 187,00 & 270,00 \\
\hline $\mathrm{SO}_{4}^{2-}$ & - & 35,07 & 30,49 & 39,02 \\
\hline $\mathrm{NH}_{4}^{+}$ & - & 0,00 & 0,00 & 0,00 \\
\hline $\mathrm{PO}_{4}{ }^{3}$ & - & 0,05 & 0,05 & 0,05 \\
\hline $\mathrm{NO}_{3}^{-}$ & 50 & 0,04 & 0,03 & 0,07 \\
\hline $\mathrm{NO}_{2}^{-}$ & - & 0,01 & 0,00 & 0,02 \\
\hline $\mathrm{K}^{+}$ & - & 35,33 & 29,46 & 45,12 \\
\hline $\mathrm{Na}^{+}$ & 200 & 32,63 & 26,32 & 45,21 \\
\hline $\mathrm{Ca}^{2+}$ & - & 89,87 & 68.48 & 102.00 \\
\hline $\mathrm{Mg}^{2+}$ & - & 18,33 & 14,50 & 21,00 \\
\hline \multicolumn{5}{|l|}{ Les métaux en mg/L } \\
\hline | & - & ND & ND & ND \\
\hline $\mathrm{Fe}$ & 0,3 & 0,54 & 0,24 & 0,76 \\
\hline $\mathrm{Mn}$ & 0,5 & 0,05 & 0,00 & 0,10 \\
\hline $\mathrm{Pb}$ & 0,01 & 0,01 & 0,01 & 0,01 \\
\hline $\mathrm{Cd}$ & 0,003 & $<0,002$ & $<0,002$ & $<0,002$ \\
\hline As & 0,01 & $<0,002$ & $<0,002$ & $<0,002$ \\
\hline $\mathrm{Cr}$ & 0,05 & 0,02 & 0,01 & 0,03 \\
\hline $\mathrm{Ni}$ & 0,02 & 0,09 & 0,02 & 0,12 \\
\hline $\mathrm{Hg}$ & 0,001 & $<0,001$ & $<0,001$ & $<0,001$ \\
\hline $\mathrm{CN}$ & 0,07 & $<0,001$ & $<0,001$ & $<0,001$ \\
\hline
\end{tabular}

\section{ND : Non Détecté}

Résultats des paramètres chimiques des eaux de source : Les cations étudiés dans les eaux de sources sont constitués des ions $\mathrm{Na}^{+}, \mathrm{K}^{+}, \mathrm{Ca}^{2+}$ et $\mathrm{Mg}^{2+}$. Parmi ceux-ci, les plus importants sont les ions $\mathrm{Ca}^{2+}$ qui représentent $51 \%$ des cations étudiés après viennent les $\mathrm{K}^{+}(20 \%)$, les $\mathrm{Na}^{+}(19 \%)$ et les $\mathrm{Mg}^{2+}(10 \%)$. Au niveau des anions, les plus importants sont $\mathrm{Cl}^{-}(69 \%)$, ensuite viennent $\mathrm{HCO}_{3}^{-}(20 \%)$ et $\mathrm{SO}_{4}{ }^{2-}(11 \%)$. Les sels nutritifs tels que $\mathrm{PO}_{4}{ }^{3}, \mathrm{NO}_{2}{ }^{-}$et $\mathrm{NO}_{3}$ - sont pratiquement inexistants dans les eaux de sources. La classification hydrochimique des eaux à partir du diagramme triangulaire de Piper montre que les eaux de MangouinYrongouin sont principalement des eaux chlorurées calciques (Figure 5). 


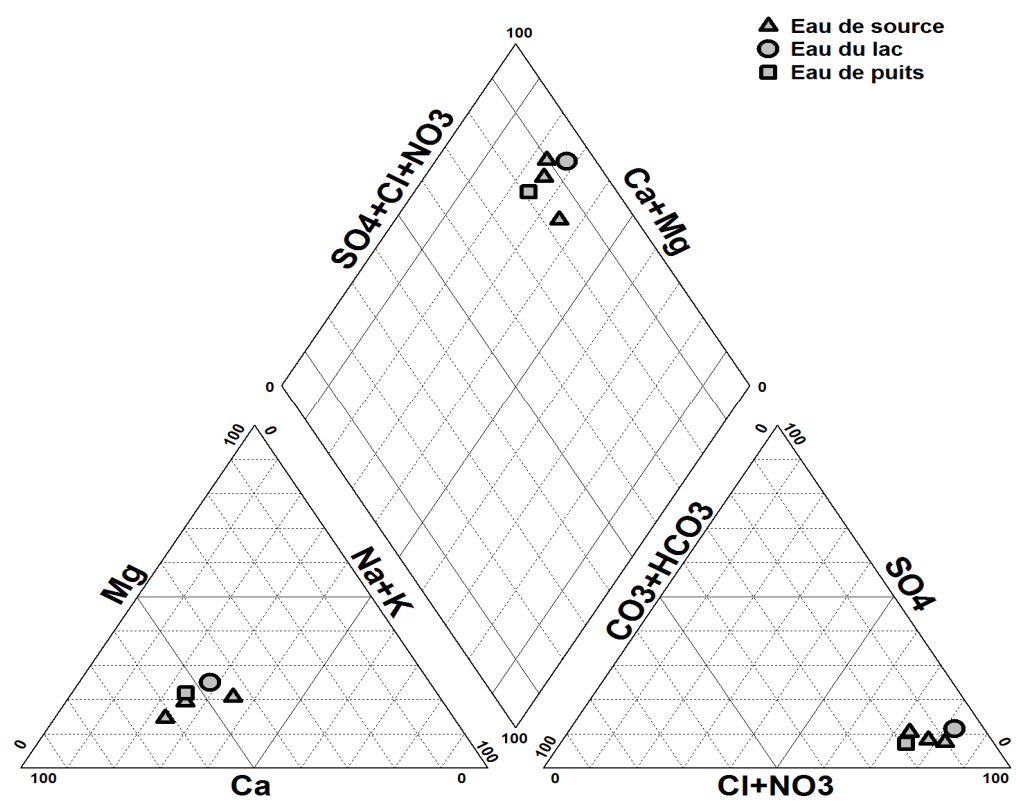

Figure 5. Classification hydrochimique des eaux de Mangouin-Yrongouin

Dans cette étude, les métaux lourds analysés sont constitués de $\mathrm{Fe}, \mathrm{Mn}, \mathrm{Pb}, \mathrm{Cd}, \mathrm{As}, \mathrm{Cr}, \mathrm{Ni}, \mathrm{Hg}$ et I (tableau 4). Parmi les métaux étudiés, on note qu'en dehors du Fe et Ni qui présentent respectivement des teneurs de 0,76 $\mathrm{mg} / \mathrm{L}$ et $0,12 \mathrm{mg} / \mathrm{L}$, supérieures à la valeur guide de l'OMS (0,3 mg/L et $0,07 \mathrm{mg} / \mathrm{L})$, tous les autres métaux sont pratiquement inexistants dans les eaux étudiées. La teneur en iode des eaux du village est largement endessous de la limite de détection, ce qui montre que les eaux du village de Mangouin-Yrongouin sont très pauvres en lode.

Tableau 4 : Teneurs en métaux lourds des eaux de Mangouin-Yrongouin

\begin{tabular}{l|c|c|c|c}
\hline Les métaux en $\mathrm{mg} / \mathrm{L}$ & Norme OMS & Moyenne & Min & Max \\
\hline $\mathrm{I}$ & & $\mathrm{ND}$ & $\mathrm{ND}$ & $\mathrm{ND}$ \\
\hline $\mathrm{Fe}$ & 0,3 & 0,54 & 0,24 & 0,76 \\
\hline $\mathrm{Mn}$ & 0,5 & 0,05 & 0,00 & 0,10 \\
\hline $\mathrm{Pb}$ & 0,01 & 0,01 & 0,01 & 0,01 \\
\hline $\mathrm{Cd}$ & 0,003 & $<0,002$ & $<0,002$ & $<0,002$ \\
\hline $\mathrm{As}$ & 0,01 & $<0,002$ & $<0,002$ & $<0,002$ \\
\hline $\mathrm{Cr}$ & 0,05 & 0,02 & 0,01 & 0,03 \\
\hline $\mathrm{Ni}$ & $\mathbf{0 , 0 7}$ & $\mathbf{0 , 0 9}$ & $\mathbf{0 , 0 2}$ & 0,12 \\
\hline $\mathrm{Hg}$ & 0,001 & $<0,001$ & $<0,001$ & $<0,001$ \\
\hline $\mathrm{CN}$ & 0,07 & $<0,001$ & $<0,001$ & $<0,001$ \\
\hline
\end{tabular}

RÉSULTATS DE L'ÉTUDE STATISTIQUE MULTIVARIÉE

Analyse en Composantes Principales (ACP) : Les valeurs propres des facteurs sont présentées dans le tableau ci-dessous. Les trois premiers facteurs représentent $83,49 \%$ de la variance exprimée (tableau
5). Ces facteurs regroupent le maximum de la variance exprimée et sont suffisants pour traduire exactement l'information recherchée. La matrice de corrélation entre les différentes variables est présentée par le tableau 6. 
Ahoussi et al... J. Appl. Biosci. 2013. Étude hydrochimique et microbiologique des eaux de source de l'ouest montagneux, Cote d'Ivoire

Tableau 5. Valeurs propres de l'ACP

\begin{tabular}{c|c|c|c|c}
\hline Facteurs & Valeur propre & \% Total de la variance exprimée & Valeur propre cumulée & Variance Cumulée \\
\hline 1 & 7,76 & 33,75 & 7,763 & 33,75 \\
\hline 2 & 5,88 & 25,55 & 13,64 & 59,30 \\
\hline 3 & 5,56 & 24,19 & 19,20 & 83,49 \\
\hline 4 & 3,80 & 16,50 & 23,00 & 100,00 \\
\hline
\end{tabular}

L'analyse des variables de l'ACP dans le plan factoriel F1-F2 est présentée par la figure 6 . Ce graphe met en

évidence trois grands regroupements des paramètres étudiés dans les points d'eau.

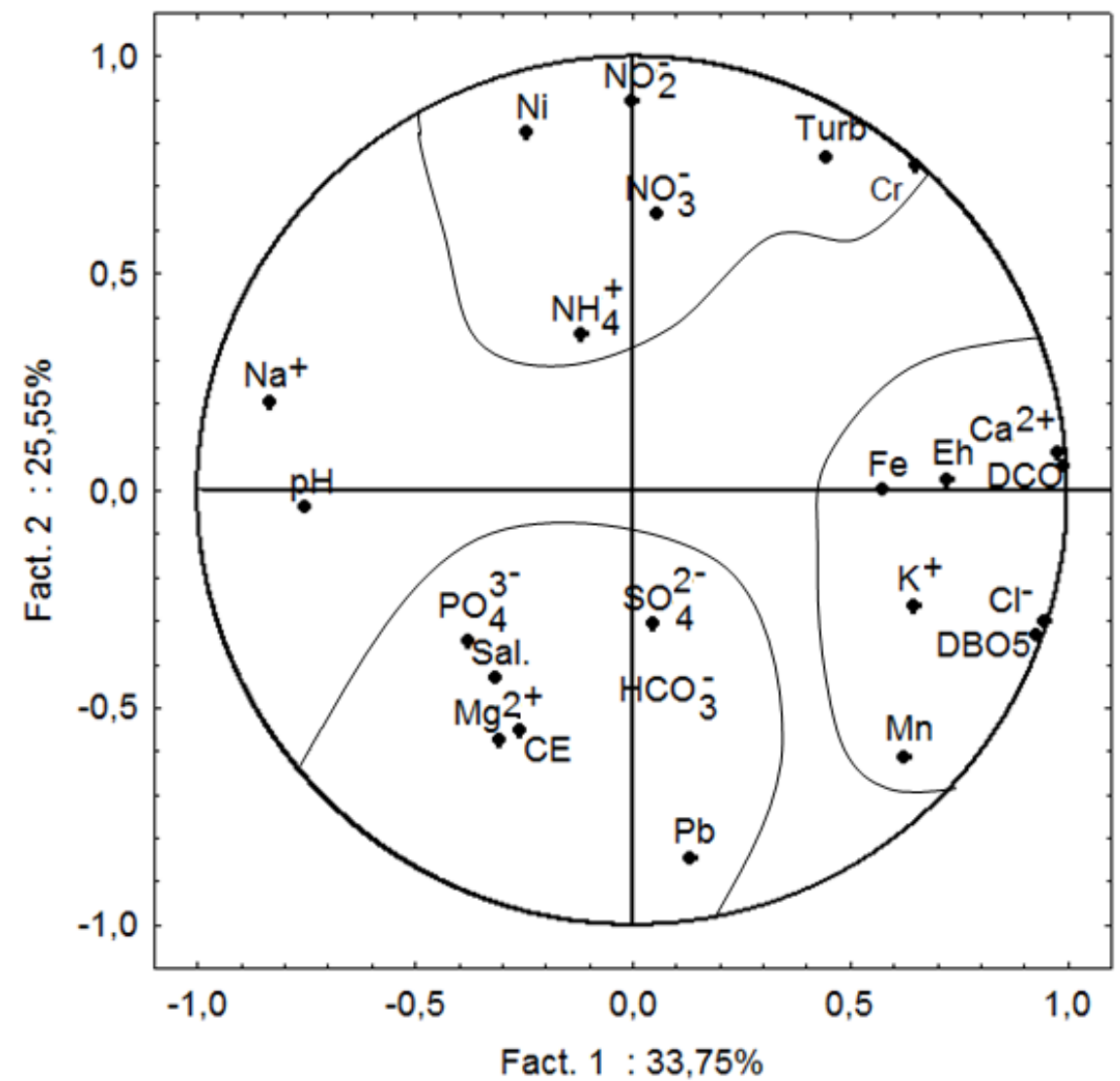

Figure 6. Analyse dans le plan factoriel F1-F2 
Ahoussi et al... J. Appl. Biosci. 2013. Étude hydrochimique et microbiologique des eaux de source de l'ouest montagneux, Cote d'Ivoire

Tableau 6 : Matrice de corrélation entre les variables

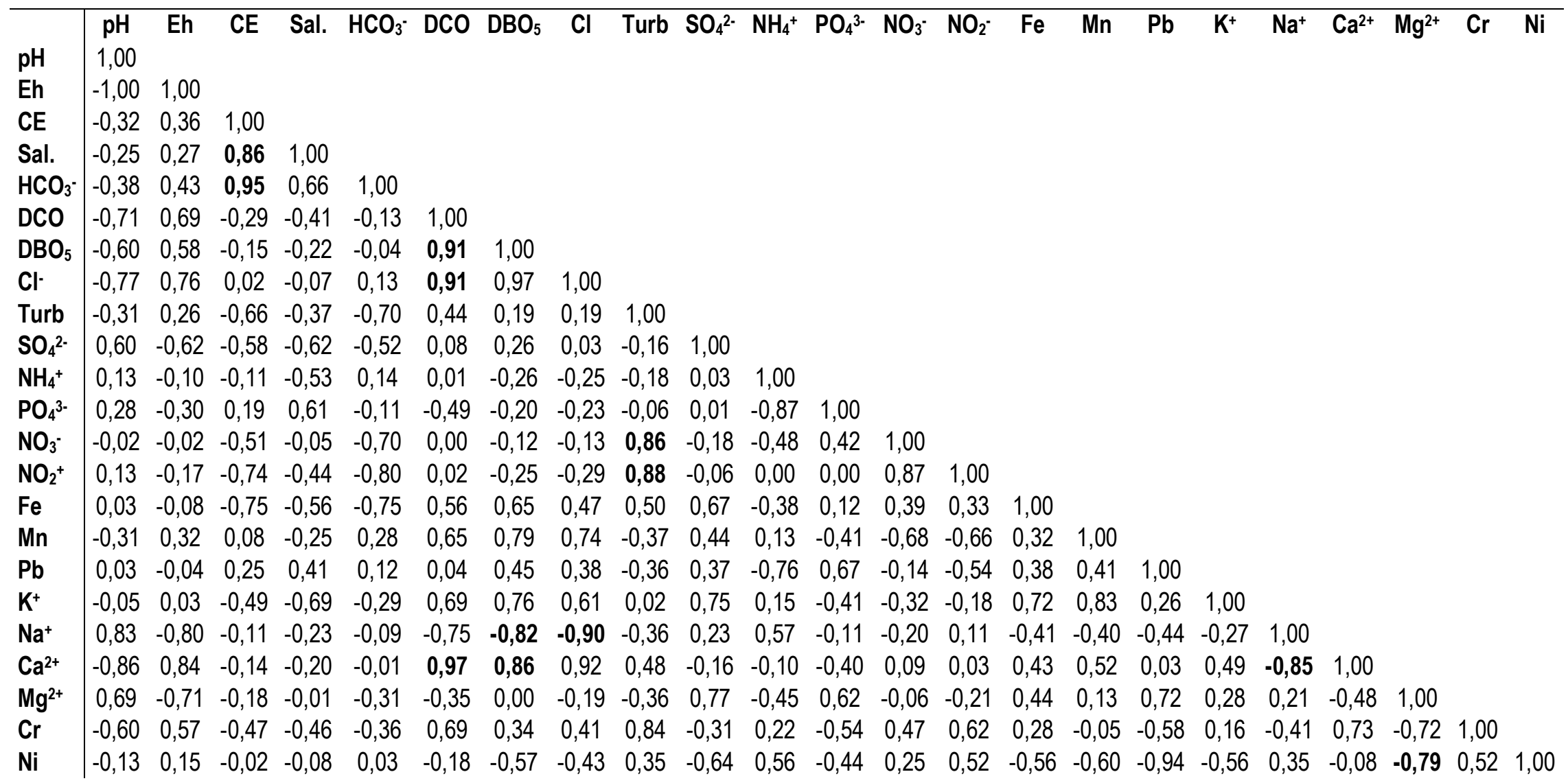




\section{Ahoussi et al... J. Appl. Biosci. 2013. Étude hydrochimique et microbiologique des eaux de source de l'ouest montagneux, Cote d'Ivoire}

Le premier regroupement qui prend en compte $\mathrm{Cl}^{-}, \mathrm{Fe}$, $\mathrm{Ca}^{2+}, \mathrm{DCO}, \mathrm{DBO}_{5}, \mathrm{Mn}, \mathrm{K}^{+}$et Eh traduit le phénomène de pluvioléssivage des sols. Le deuxième regroupement contient $\mathrm{Mg}^{2+}, \mathrm{PO}_{4}^{3-}$, Sal., $\mathrm{SO}_{4}{ }^{2-}, \mathrm{HCO}_{3}{ }^{-}, \mathrm{Pb}, \mathrm{Sal}, \mathrm{CE}$, $\mathrm{HCO}_{3}-$ met en évidence une minéralisation des eaux liée au contact eau-roche (minéralisation temps de séjour). Enfin, le troisième qui prend en compte $\mathrm{NH}_{4}{ }^{+}, \mathrm{Ni}$, Turb., $\mathrm{Cr}, \mathrm{NO}_{2}$ - et $\mathrm{NO}_{3}-$ montre une minéralisation anthropique issue des activités agricoles. En somme, les différents regroupements étudiés montrent que la minéralisation des eaux étudiées est liée à trois phénomènes importants d'éléments minéraux. II s'agit du phénomène de pluviolessivage, de la minéralisation issue des activités anthropiques et de la minéralisation par le contact eauroche. Le dendrogramme (Figure 7) issu de la Classification Hiérarchique Ascendante (CHA) a mis en évidence trois principaux regroupements des variables. La première famille qui prend en compte $\mathrm{Pb}, \mathrm{Cl}^{-}, \mathrm{CE}$, $\mathrm{HCO}_{3}{ }^{-}, \mathrm{Ca}^{2+}$, DCO et Eh traduit une minéralisation liée au phénomène de pluviolessivage des sols. Le second regroupement qui est constitué de $\mathrm{Mg}^{2+}, \mathrm{Na}^{+}, \mathrm{SO}_{4}{ }^{2+}, \mathrm{K}^{+}$et $\mathrm{DBO}_{5}$ explique la minéralisation temps de séjour (contact eau-roche). Le troisième est constitué de $\mathrm{pH}$, Turb., Sal., $\mathrm{Ni}, \mathrm{Fe}, \mathrm{PO}_{4}{ }^{3-}, \mathrm{NH}_{4}{ }^{+}, \mathrm{NO}_{3}{ }^{-}, \mathrm{NO}_{2}{ }^{-}$et $\mathrm{Cr}$ met en évidence une minéralisation liée aux apports des sels nutritifs d'origine anthropique.

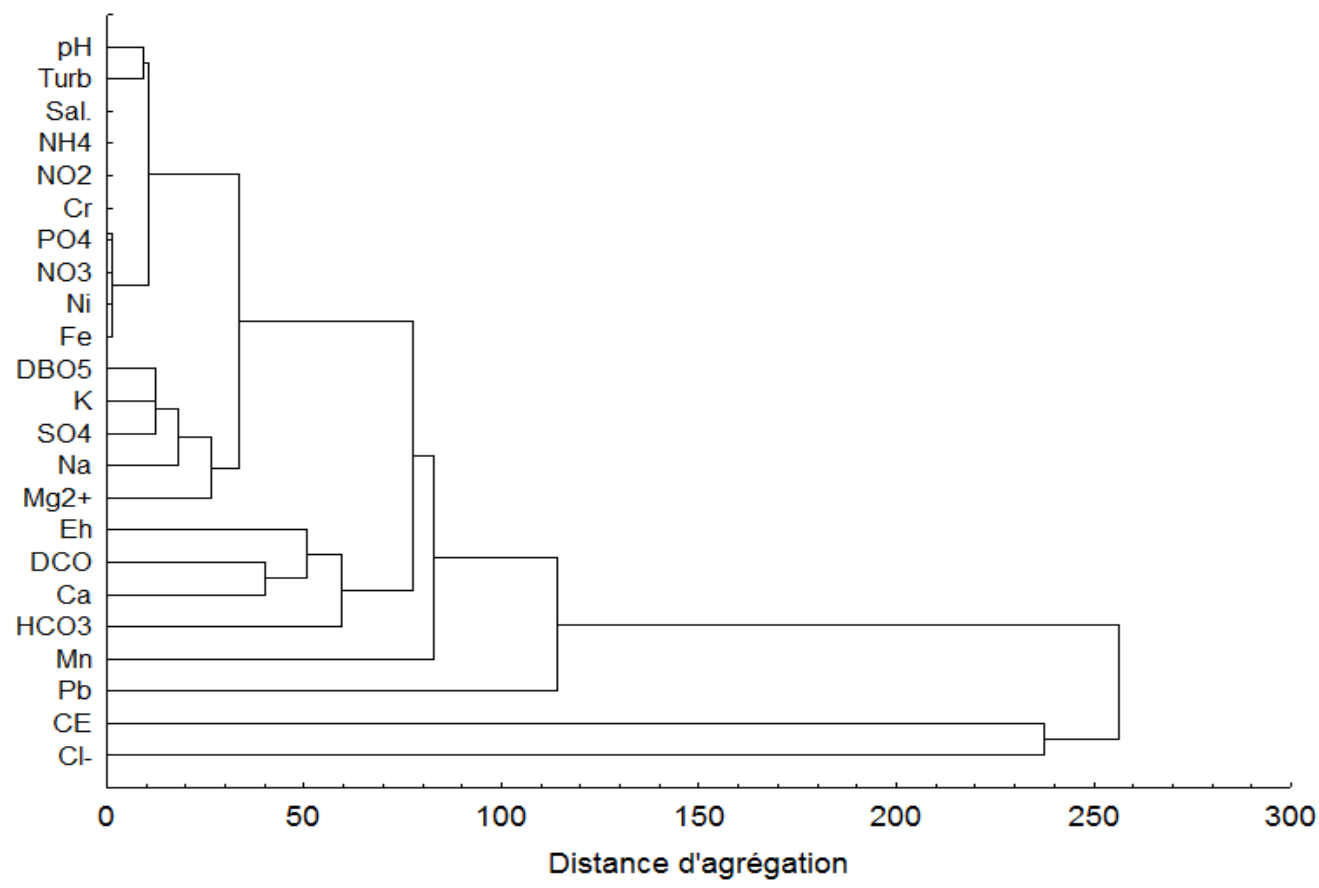

Figure 7. Dendrogramme des paramètres physico-chimiques des eaux de Mangouin-Yrongouin

La classification des points d'eau selon leur ressemblance dans le cadre d'un suivi environnemental des points d'eau étudiés est donnée par la figure 8. Ce dendrogramme met en évidence deux regroupements des points d'eau étudiés. Le premier regroupement prend en compte les eaux de source (E1, E2, E3 et E5) et le second contient les eaux souterraines (E4). Pour le suivi de la qualité des eaux, il est important de choisir un point de suivi dans chaque regroupement. Ainsi, deux points d'eau sont nécessaires (un puits et une source). 


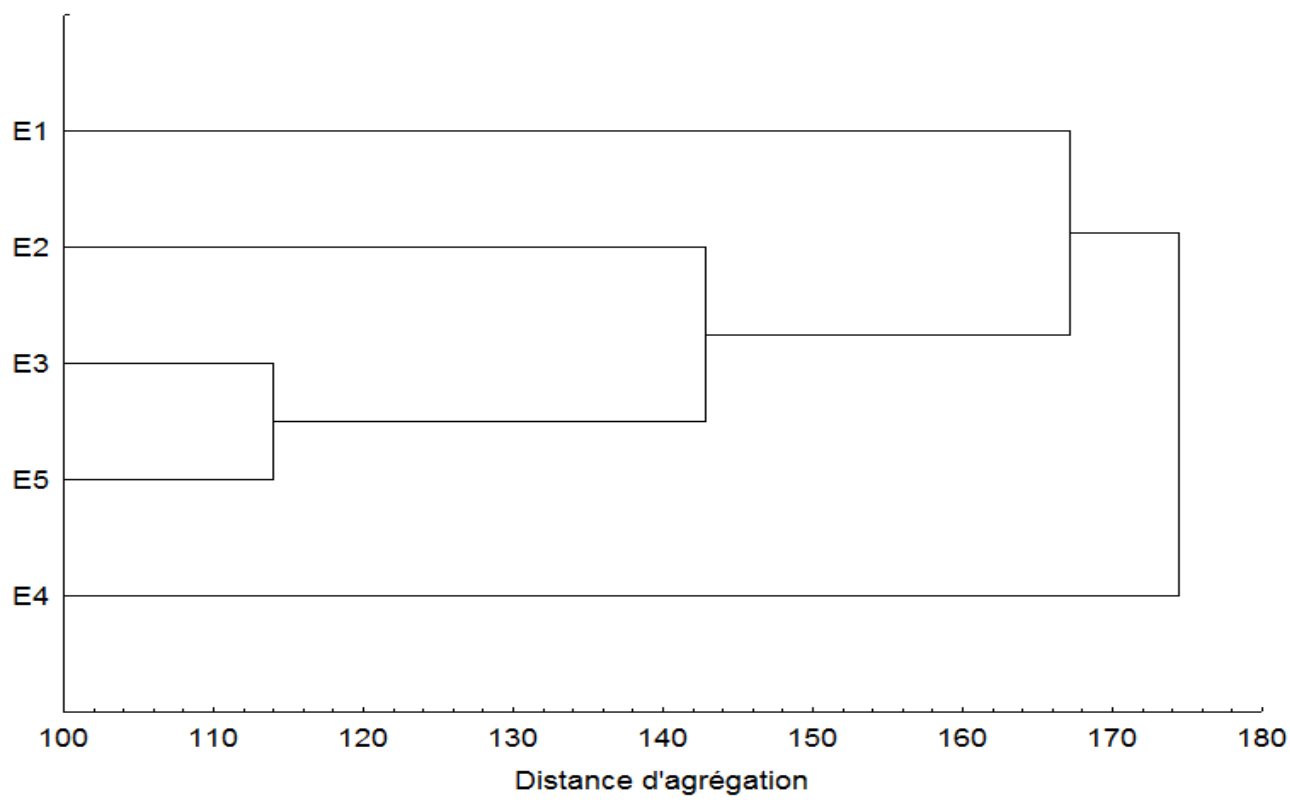

Figure 8. Dendrogramme pour la classification des points d'eau de Mangouin

Résultats des analyses microbiologiques des eaux : Les résultats des analyses microbiologiques indiquent la présence des coliformes fécaux principalement l'Escherichia Coli dans l'eau du lac et l'eau de puits. La totalité des eaux échantillonnées contiennent des clostridium perfringens et des coliformes totaux (tableau 7).

Tableau 7. Résultats des analyses microbiologiques des eaux de Manguion-Yrongouin

\begin{tabular}{l|l|c|c|c|c|c|c|c}
\hline & & & & Source 1 & Lac 2 & Source 3 & Puits 4 & Source ${ }^{\circ} \mathbf{5}$ \\
\hline Paramètres & Méthodes & Unités & Critères & Résultats & Résultats & Résultats & Résultats & Résultats \\
\hline $\begin{array}{l}\text { Dénombrement } \\
\text { des coliformes } \\
\text { totaux }\end{array}$ & NF V08-50 & ufc/100ml & $>1 / 100 \mathrm{ml}$ & 0 & 30000 & 1000 & 2100 & 6000 \\
\hline $\begin{array}{l}\text { Dénombrement } \\
\text { des Escherichia } \\
\text { Coli }\end{array}$ & $\begin{array}{l}\text { AFNOR } \\
\text { BRD 07/1- } \\
07 / 93\end{array}$ & ufc/100ml & $>1 / 100 \mathrm{ml}$ & 0 & 500 & 0 & 400 & 0 \\
\hline $\begin{array}{l}\text { Dénombrement } \\
\text { des clostridium } \\
\text { perfringens }\end{array}$ & $\begin{array}{l}\text { NF -V-08- } \\
056\end{array}$ & ufc/100ml & $>1 / 20 \mathrm{ml}$ & 100 & 1500 & 100 & 100 & 100 \\
\hline
\end{tabular}

Les analyses révèlent la présence de coliformes fécaux dans les eaux du lac de Granoci et dans les eaux de puits du village, avec respectivement des teneurs de 500 et $400 \mathrm{ufc} / 100 \mathrm{ml}$. Dans cette localité, toutes les eaux étudiées contiennent des fortes teneurs de Clostridiums

\section{DISCUSSION}

Les analyses in situ réalisées sur les eaux de source de la localité de Mangouin-Yrongouin montrent que les eaux sont faiblement minéralisées, avec une conductivité électrique moyenne de $152,90 \mu \mathrm{S} / \mathrm{cm}$. Les eaux sont
Perfringens. Celles-ci varient respectivement de 100 à $1500 \mathrm{ufc} / 20 \mathrm{ml}$. La présence de ces micro-organismes dans l'eau traduit une pollution fécale d'origine humaine, ce qui rend compte de l'influence des activités anthropiques sur la qualité des eaux de source.

acides, avec un pH qui varie de 4,85 à 6,01 , pour une moyenne de 5,34. L'acidité des eaux constitue un des caractères essentiels des eaux en Côte d'Ivoire en général et dans la région de l'ouest montagneux en 


\section{Ahoussi et al... J. Appl. Biosci. 2013. Étude hydrochimique et microbiologique des eaux de source de l'ouest montagneux, Cote d'Ivoire}

particulier (Biémi, 1992). En effet, selon Tapsoba (1995), Matini et al. (2009) et Ahoussi et al. (2010), l'acidité des eaux en zone tropicale humide est principalement liée à la décomposition de la matière organique végétale, avec la production de $\mathrm{CO}_{2}$ dans les premières couches du sol. La présence dans l'eau de $\mathrm{CO}_{2}$ provenant des sols facilite l'hydrolyse des minéraux silicatés et la formation des ions $\mathrm{HCO}_{3}$ : En effet, les résultats de l'analyse multivariée montrent que dans la région l'hydrolyse acide des roches constitue un phénomène important dans la minéralisation des eaux. La région étant une zone forestière agricole, les apports de ces activités dans la minéralisation sont marqués par l'enrichissement des eaux en sels nutritifs. Les eaux étudiées contiennent les coliformes fécaux du type Eschérichia coli et des clostridium perfringens. Cela montre que les eaux sont soumises à une pollution microbiologique d'origine humaine (Ahoussi et al., 2012b). En effet, la localité de Mangouin-Yrongouin, comme dans la quasi-totalité des villages de la Côte d'Ivoire est dépourvue d'un système d'assainissement adéquat. Les latrines étant inexistantes, les populations font généralement leur besoins personnels dans la nature. Dans cette localité, les pluviométries sont très abondantes et les eaux de ruissellement érodent les sols et les déposent dans les cours d'eau et les sources. Lors de cette étude, les observations de terrain ont mis en évidence la présence d'excréments humains aux alentours de différents points d'eaux. La présence de coliformes fécaux dans les eaux confirme l'influence des

\section{CONCLUSION}

Les études réalisées dans la localité de MangouinYronguin ont permis une caractérisation hydrogéochimique et microbiologique des ressources en eau. Cette étude montre que dans la zone d'étude, le niveau statique de la nappe phréatique varie de $0,48 \mathrm{~m}$ à 4,52 $\mathrm{m}$, avec une moyenne de 1,24 $\mathrm{m}$. Les eaux souterraines sont peu profondes et très vulnérables à la pollution d'origine anthropique. Les analyses physicochimiques des eaux révèlent que celles-ci se classent en un seul hydrofaciès constitué par les eaux chlorurées calciques. La minéralisation des eaux de la zone d'étude est contrôlée par plusieurs phénomènes dont le pluvioléssivage des sols, l'hydrolyse acide des roches

\section{REFÉRENCES BIBLIOGRAPHIQUES}

Ahoussi K. E., Soro N., Soro G., Lasm T., Oga M. S., Zadé S. 2008. Groundwater Pollution in Africans Biggest Towns: Case of the Town of Abidjan (Côte d'Ivoire). European Journal of Scientific Research, 20(2) : 302-316. activités anthropiques du village Mangouin-Yrongouin sur celles-ci. Boutin (1993) explique la présence des coliformes fécaux dans les eaux de puits par une contamination d'origine fécale et donc la possibilité que les germes pathogènes dangereux soient présents dans l'eau. En Côte d'Ivoire, l'origine fécale de la pollution des ressources en eau a été mise en évidence par les auteurs tels qu'Ahoussi et al. (2009, 2011, 2012a et 2012b), dans les eaux de surface et les eaux souterraines des localités d'Abidjan, de Kossihouen et de N'zianouan. Au Sud du Kenya, Ombaka et Gichumbi (2012) ont mis en évidence une forte pollution microbiologique d'origine anthropique dans les eaux de la rivière Ruguti au Meru. L'influence des activités anthropiques sur la qualité des eaux s'observe également dans la région de Baikunthpur, le District de Rewa, Madhya et Pradesh en Inde par Tiwari et Umesh Mishra (2012). Dans la zone d'étude, l'origine des métaux lourds dans l'eau est essentiellement liée à la nature des formations géologiques. En effet, l'ouest montagneux de la Côte d'Ivoire est une zone riche en ressources minières, avec la présence de gisements de fer, de nickel et de cobalt. Ces éléments se retrouvent dans les eaux de source et les eaux souterraines après altération de la roche mère. Les eaux de la localité de MangouinYrongouin sont pauvres en lode. Cela constitue une particularité des eaux de l'ouest montagneux de la Côte d'Ivoire (Biémi, 1992).

sous-jacente et les activités anthropiques de la zone. Les analyses microbiologiques montrent également la présence de coliformes fécaux (Escherichia coli), et de Clostridiums Perfringens et des coliformes totaux dans les eaux. La présence de ces microorganismes dans les eaux de Mangouin-Yrongouin montre que celles-ci sont exposées à une pollution microbiologique fécale d'origine humaine. Cette pollution détériore gravement la qualité physico-chimique et microbiologique des eaux. Elle constitue un risque sanitaire pour les populations de ladite localité qui sont largement tributaires de ces eaux pour leur besoins.

Ahoussi K. E., Soro N., Soro G., Oga M. S., Zadé S. 2009. Caractérisation de la qualité physicochimique et bactériologique des eaux de puits de la ville d'Abidjan (Côte d'Ivoire). Africa Geoscience Review, 16(3): 199-211. 


\section{Ahoussi et al... J. Appl. Biosci. 2013. Étude hydrochimique et microbiologique des eaux de source de l'ouest montagneux, Cote d'Ivoire}

Ahoussi K. E., Soro N., Koffi Y. B., Soro G., Biémi J. 2010. Origine de la minéralisation des eaux des aquifères discontinus sous couvert forestier de la zone Sud de la Côte d'Ivoire: cas de la région d'Abidjan-Agboville. International Journal of Biological and Chemical Sciences, 4(3): 782797.

Ahoussi K. E., Oga Y. M. S., Koffi Y. B., Kouassi A. M., Soro N., Biémi J. 2011. Caractérisation hydrogéochimique et microbiologique des ressources en eau du site d'un Centre d'Enfouissement Technique (CET) de Côte d'Ivoire : cas du CET de Kossihouen dans le District d'Abidjan (Côte d'Ivoire). International Journal of Biological and Chemical Sciences, 5(5): 2114-2132.

Ahoussi K. E., Koffi Y. B., Kouassi A. M., Soro G., Soro N., Biémi J. 2012a. Étude des caractéristiques chimiques et microbiologiques des ressources en eau du bassin versant du N'zi : cas de la commune de N'zianouan (Sud de la Côte d'Ivoire). International Journal of Biological and Chemical Sciences, 6(4): 1854-1873

Ahoussi K. E., Koffi Y. B., Kouassi A. M., Soro G., Soro N., Biémi J. 2012b. Caractérisation PhysicoChimique et Bactériologique des Ressources en eau des Localités Situées Aux Abords de la lagune Ébrié dans la commune de Marcory (District d'Abidjan, Côte Ivoire) : cas du village d'Abia Koumassi. European Journal of Scientific Research, Vol. 89 № 3 : 359-383

Biémi J. 1992. Contribution à l'étude géologique, hydrogéologique et par télédétection des bassins versants Subsahéliens du socle précambrien d'Afrique de l'Ouest : Hydrostructurale, hydrochimie et isotopie des aquifères discontinus des sillons et aires granitiques de la Haute Marahoué (Côte d'Ivoire). Thèse d'Etat, Université Nationale de Côte d'Ivoire, p. 480.

Boutin C. 1993. L'eau des nappes phréatiques superficielles, une richesse naturelle mais vulnérable. L'exemple des zones rurales du Maroc. Sciences de l'eau, 6(3) : 357-365.

Camil J, Tempier. P, vachette. C. 1984. Schéma pétrographique et structural et chronologique des formations archéennes de la région de Man (Côte d'Ivoire). Leur rôle dans la cratonisation de l'Ouest Africain. Geol. Afric. Ed. Tervuren, 10 p.
Coulibaly A., Mondé S., Wognin V. A., Aka K. 2008. State of anthropic pollution in the estuary of Ebrié lagoon (Côte d'lvoire) by analysis of the metal elements traces. European Journal of Scientific Research, 19(2): 372-390.

DJRO. S. C. (1998): Evolution tectono-métamorphiques des gneiss granulitiques archéens du secteur de Biankouma. Thèse de Doctorat d'Etat de l'Université de Cocody, p.171.

Güler C., Thyne G. D., Mccray J. E., Tuner A. K. 2002. Evaluation of graphical and multivariate statistical methods for classification of water chemistry data. Hydrogeology Journal (10): 455-474

Kouamelan A. N. 1996. Géochronologie et géochimie des formations Archéennes et Protérozoïques de la dorsale de Man en Côte d'lvoire. Implications pour la transition Archéenneprotérozoique. Thèse. Doct. Es Sc Nat. Univ. Rennes 1. $277 p$.

Kouzana L., Ben Mammou A., Gaaloul N. 2007. Intrusion marine et salinisation des eaux d'une nappe phréatique côtière (Korba, Cap-Bon, Tunisie). Geo-Eco-Trop., 31 : 57-70.

Matini L., Moutou J.M., Kongo-Mantono M.S. 2009. Evaluation hydrochimique des eaux souterraines en milieu urbain au Sud-Ouest de Brazzaville, Congo. Afrique Science 05(1) : 82-98.

Ombaka O., Gichumbi 2012. Water quality assessment of Ruguti River in Meru South, Kenya. International Journal of Water and Environmental Engineering, Vol.4 (12): 404-414.

OMS 2008. Guidelines for Drinking-water Quality. Third edition, incorporating the first and second Addenda, Recommendations, Geneva, Volume $1, \mathrm{p} 515$.

Oga M. S., Lasm T., Yao T. K., Soro N., Saley M. B., Kouassi D., Gnamba F. 2009. Caractérisation chimique des eaux des aquifères de Fracture: Cas de La Région de Tiassalé en Côte d'Ivoire. European Journal of Scientific Research, 31(1):72-87.

Saley M. B. 2003. Cartographie thématique des aquifères de fissures pour l'évaluation des ressources en eau. Mise en place d'une nouvelle méthode d'extraction des discontinuités images et d'un SIHRS pour la région semi-montagneuse de Man (Nord-Ouest de la Côte d'Ivoire). Thèse de Doctorat d'Université de Cocody-Abidjan, $209 \mathrm{p}$.

Soro N., Ouattara L., Dongo K., Kouadio K. E., Ahoussi K. E., Soro G., Oga Y. M.-S., Savane I., Biémi J. 
2010. Déchets municipaux dans le District d'Abidjan en Côte d'Ivoire : sources potentielles de pollution des eaux souterraines. International Journal of Biological and Chemical Sciences, 4 (6): 2203-2219.

Tiwari R. N., Umesh Mishra 2012. Groundwater quality and pollution potential studies around Baikunthpur area, Rewa District, Madhya Pradesh, India. International Journal of Water and Environmental Engineering, Vol.4 (12): 377385.

Tapsoba S. A. 1995. Contribution à l'étude Géologique et Hydrogéologique de la région de Dabou (Sud de la Côte d'Ivoire) : Hydrochimie, Isotopie et Indice de vieillissement des eaux souterraines, Thèse de $3^{\text {è }}$ cycle de l'Université Nationale de Côte d'Ivoire, p. 200.

Thilagavathi R.,Chidambaram S., Prasanna M. V. Thivya C., Singaraja C. 2012. A study on groundwater geochemistry and water quality in layered aquifers system of Pondicherry region, southeast India. Applied. Water Science. 2: 253269.

Yermani M, Zouari K, Michelot JL., Mamou A, Moumni L. 2003. Approche géochimique du fonctionnement de la nappe profonde de Gafsa Nord (Tunisie centrale). Journal des Sciences Hydrologiques, 48(1): 95-108.

Yidana S.M., Ophori D., Banoeng-Yakubo B. 2008. A multivariate statistical analysis of surface water chemistry data - The Ankobra Basin, Ghana. Journal of Environmental Management, 86: 8087. 